\title{
Abelian Cascade Dynamics in Bootstrap Percolation
}

\author{
S. S. Manna* \\ Satyendra Nath Bose National Centre for Basic Sciences \\ Block-JD, Sector-III, Bidhannagar, Calcutta 700091, India
}

$\underline{\text { Abstract }}$

The culling process in Bootstrap Percolation is Abelian since the final stable configuration does not depend on the details of the updating procedure. An efficient algorithm is devised using this idea for the determination of the bootstrap percolation threshold in two dimension which takes $L^{2}$ time compared to the $L^{3} \log L$ in the conventional method. A generalised Bootstrap Percolation allowing many particles at a site is studied where continuous phase transitions are observed for all values of the threshold parameter. Similar results are also obtained for the continuum Bootstrap Percolation model.

PACS numbers : 05.50.+q, 64.60.Ak, 64.60.-i, 07.05.Kf

*e-mail: manna@boson.bose.res.in 
In the Bootstrap Percolation model (BPM) sites of a regular lattice are occupied randomly with a probability $p[1]$. A culling condition is imposed: Occupied sites having fewer than $m$ occupied neighbours are successively removed. Finally the lattice may be totally empty or may have a specific distribution of occupied sites. Beyond a threshold value $p_{c}^{*}(m)$ of $p$ the stable configuration $(\mathrm{SC})$ in an infinitely large system has a spanning "infinite" cluster with probability 1 [1-3].

Motivation behind studying BPM originates in the properties of some magnetic materials like $T b_{c} Y_{1-c} S b$ where a strong competition exists between the exchange and crystal field interactions. Due to the exchange interaction the atomic spins tend to allign where as the crystal field tend to suppress the magnetic moments [4]. In such a pure system where the effect of exchange interaction is just sufficient to overcome the effect of the crystal field, the result of introducing non-magnetic impurities may be significant. A spin with a magnetic moment if surrounded by too many non-magnetic neighbours may become non-magnetic. Therefore even at a low temperature, as the impurity concentration is gradually increased, at a certain stage the magnetic order may get destroyed [1].

In BPM the global connectivity of the stable configuration is tested with increasing concentration of impurity. By definition for all values of $m, p_{c}^{*}(m) \geq p_{c}$, the ordinary percolation threshold [5]. Applying BP rule on a random initial configuration at $p \rightarrow p_{c}+$, nothing is culled for $m=0$, the isolated occupied sites are culled for $m=1$ and the dangling chain of sites are also culled for $m=2$. In these cases the connectivity remains intact which implies $p_{c}^{*}(m)=p_{c}$ for $m=0,1$ and 2 in any arbitrary lattice.

A cluster of occupied sites, in which every site has at least $m$ neighbours, is called an $m$-cluster [2]. The SC contains only $m$-clusters. An isolated cluster in a dimensional hypercubic lattice always has some convex corners on the surface with $d$ neighbours. Therefore, for $m \geq d+1$ in the hypercubic lattices, the SC has only the infinite $m$-clusters. For example, for $m=3 \mathrm{BPM}$ on the square lattice, the $\mathrm{SC}$ is a single infinite 3-cluster with rectangular holes [3]. Schonmann has shown that for $m \geq d+1$ in hypercubic lattices $p_{c}^{*}(m)=1[6]$. This was also predicted from Straley's theory of 'large void instabilities' [7]. 
The bootstrap percolation probability $Q_{m}(p)$ is the probability that a site belongs to the infinite cluster. For those values of $m$ where $p_{c}^{*}(m)=p_{c}$, the transition is continuous like percolation. For other values of $m \geq d+1$ first order transitions are observed. As $p \rightarrow p_{c}^{*}(m)+$, a SC on further removal of even a single site finally becomes empty showing a discontinuous drop in $Q_{m}(p)$ indicating a first order transition.

Numerical estimation of $p_{c}^{*}(m)$ turns out to be a difficult task. For a system of size $L$ an effective threshold $p_{c}^{*}(m, L)$ is obtained after configuration averaging which slowly converges to $p_{c}^{*}(m)$ in the limit of $L \rightarrow \infty$. For example, for $m=d+1$ on hypercubic lattices, Schonmann has shown that the asymptotic value is approached with a scaling correction $1 / \log ^{d-1} L[6]$. Using the definition, BPM is best studied by the "multi-spin coding technique" where occupied and vacant sites are represented by the two states of a bit [8-9]. A $B$ bit integer word storing $B$ sites is updated by a logical equation involving the neighbouring words. For example, for $m=3 \mathrm{BPM}$ on the square lattice, if the four neighbouring words are $w_{1}, w_{2}, w_{3}$ and $w_{4}$, the central word $w_{c}$ is updated as:

$$
w_{c}=\operatorname{not}\left[\left(\bar{w}_{1}+\bar{w}_{2}\right) *\left(\bar{w}_{3}+\bar{w}_{4}\right)+\bar{w}_{1} * \bar{w}_{2}+\bar{w}_{3} * \bar{w}_{4}\right]
$$

where $\bar{w}=\operatorname{not}(w)$ and + and $*$ symbols denote the or and and operations. The average number of iteration steps necessary to reach the SC at $p_{c}^{*}$ increases linearly with $L$. Again, the $p_{c}^{*}$ is estimated by successively halving certain interval $p^{\text {high }}$ and $p^{\text {low }}$ which needs around $\log L$ number of different runs. Therefore ordinarily the computer effort increases as $L^{3} \log L$ near $p_{c}^{*}$ for a two dimensional system of size $L$.

We first observe that given a particular initial configuration the $\mathrm{SC}$ in any $\mathrm{BPM}$ is always the same irrespective of whether a parallel, sequential or a mixed updating procedure is used. The 'occupation number configuration'(ONC) is described by defining at every site $i, s_{i}=1$ (occupied) or 0 (vacant) and the number of occupied neighbours as the "neighbour number'(NN) $n_{i}=\Sigma_{j}^{n n} s_{j}$ where $j$ runs over the nearest neighbours. Then the culling of a site can be written as:

$$
\text { If } \quad n_{i}<m, \text { then } s_{i} \rightarrow 0, \quad n_{i} \rightarrow n_{o}
$$


and $\quad n_{j} \rightarrow n_{j}-1$

where $j$ runs over the unculled occupied neighbours only and $n_{o}$ is any fixed large number. Now consider two arbitrary sites $i$ and $j$ which are to be culled. If we first cull the site $i$ and then the site $j$, then for some sites the NNs decrease by one and for the sites which are common neighbours of both $i$ and $j$ the NNs decrease by two. We see that the new ONC and NNC are exactly the same if the sites $i$ and $j$ were culled in the reverse order. Therefore the resulting configuration is symmetric under the exchange of the sites $i$ and $j$. If we apply this argument repeatedly we see that at the end of the BP process the same ONC and NNC appear irrespective of the choice of sequence in which the different sites are culled. This process is reminiscent of the Abelian sandpile model (ASM) of Self-Organized Criticality where same SC is obtained irrespective of the sequence of the sand grain additions [10]. Hence we shall call our culling process as Abelian.

A cascade of culling sites can be grown from a single culled site. A site $i$ with $n_{i}<m$ is selected on an arbitrary ON configuration and culled. It may result in some more sites with $n_{j}<m$ in the neighbourhood and are culled again. This creates other sites in the further neighbourhood ready for culling. In this way a cascade of culling sites continues which stops when no further site can be culled. We call the set of sites culled in the cascade as the 'culled cluster' (CC) analogous to the 'avalanche cluster' in ASM [11].

This idea greatly helps us to find out the SC. The difference between an ON configuration and its SC is a set of clusters of culled sites. Each such cluster is in general a combination of many CCs. Since the culling process is Abelian it does not matter in which sequence these CCs are culled. Therefore given an ON configuration, we try to grow CCs from all sites sequentially one after the other. The remaining unculled sites constitute the SC. Since a site can be culled at most once, the computer effort is proportional to the area $L^{2}$ of the system compared to $L^{3}$ in the ordinary method.

The BP threshold $p_{c}^{*}(m)$ can also be obtained starting from a fully occupied lattice and then deleting the sites one after another. After deleting a site the corresponding SC 
is checked for percolation. The process is continued till the SC ceases to percolate. This process however becomes much easier on using the Abelian property. The SC after deleting $(n+1)$ sites is obtained by removing the $(n+1)$-th site from the SC after deleting $n$ sites and then growing a CC from the deleted $(n+1)$-th site. If $f_{c}^{*}$ is the fraction of deleted sites when the SC just stops percolating, then $p_{c}^{*}=1-f_{c}^{*}$ is the $\mathrm{BP}$ threshold for this configuration (Fig.1). Therefore our algorithm takes $L^{2}$ time compared to $L^{3} \log L$ in the ordinary method. In ordinary method, huge time is wasted in repeated update attempts to already culled sites. In our algorithm, only the culling sites are updated by growing one CC after another. Another method, called "rectangle" algorithm has some indication to be faster than ordinary BP algorithm for large systems and near the percolation threshold [12].

Here we introduce a generalised BPM by allowing a site to be occupied up by more than one particle. On a square lattice, sites are filled up randomly to an average density of $\rho$ particles per site so that $s_{i}$ is the number of particles at the site $i$. Here the neighbourhood includes the site itself and $\mathrm{NN}$ is defined as $n_{i}=\Sigma_{j}^{n n} s_{j}+s_{i}-1$. Now, if $n_{i}<m$, we delete all particles at $i$ as: $s_{i} \rightarrow 0$ and the NN at $i$ is raised to $n_{i} \rightarrow n_{o}$ whereas the NNs of the neighbouring unculled sites are decreased as $n_{j} \rightarrow n_{j}-s_{i}$. To obtain the SC, CCs are grown sequentially from all the lattice sites satisfying the culling condition $n_{i}<m$. We use the Hoshen Kopelman algorithm [13] to test if the SC has an infinite percolation cluster.

The percolation thresold $\rho_{c}^{*}(m)$ is the minimum value of the average initial density $\rho$ beyond which an infinite cluster exists with probability 1 in the SC. The percolation probability $Q_{m}(\rho)$ is the fraction of particles that survive on the infinite percolation cluster. Like BPM on the Cayley tree [1], our results indicate a gap as: $Q_{m}(\rho)-Q_{m}\left(\rho_{c}^{*}(m)\right) \sim(\rho-$ $\left.\rho_{c}^{*}(m)\right)^{\beta}$ where, $Q_{m}\left(\rho_{c}^{*}(m)\right)=\operatorname{Lim}_{\rho \rightarrow \rho_{c}^{*}(m)} Q_{m}(\rho)$ in the limit $L \rightarrow \infty$. However, according to the scaling theory [5], for finite systems the scaling variable should be $L / \xi$, where $\xi=$ $\left(\rho-\rho_{c}^{*}(m)\right)^{-\nu}$ is the correlation length. Consequently the percolation probability should behave as: $Q_{m}(\rho)-Q_{m}\left(\rho_{c}^{*}(m)\right)=L^{-\beta / \nu} F\left[\left(\rho-\rho_{c}^{*}(m)\right) L^{1 / \nu}\right]$ where the scaling function $F(x) \rightarrow x^{\beta}$ for large $L$. For $m=2$ we calculate the $Q_{2}(\rho)$ as a function of $\rho$ on system sizes up to $L=1024$. The curves for different systems are found to be continuous and pass 
through a fixed point with coordinates $\left(\rho_{c}^{*}(2), Q_{2}\left(\rho_{c}^{*}(2)\right)\right)=(0.899,0.179)$ (Fig. 2). In Fig. 3, $\left(Q_{2}(\rho)-Q_{2}\left(\rho_{c}^{*}(2)\right)\right) L^{0.075}$ is plotted with $\left(\rho-\rho_{c}^{*}(2)\right) L^{0.75}$ and an excellent collapse of the data on a continuous curve is observed. We conclude that $\nu \approx 4 / 3$ and $\beta \approx 0.10$ compared to the percolation values $4 / 3$ and $5 / 36$ in the ordinary percolation [5].

Since the number of particles at a site is not bounded therefore even the isolated sites with $s_{i}>m+1$ cannot be culled. Similarly, a pair of adjacent sites $i$ and $j$ with $s_{i}+s_{j} \geq m+1$ also survives. Thus the SC at any $\rho$ contains many islands. As we increase $\rho$, size of these islands increase and they merge into one another. This merging process is same as in ordinary percolation, hence we expect the transition to be continuous, and in the same universality class.

Suppose now that every sites has exactly $\rho$ particles. Then $\rho_{c}^{*}(m)=(m+1) / 5$. However, in a random process a site has on the average $\rho$ particles with a fluctuation around $\sqrt{\rho}$. Therefore even if $\rho=(m+1) / 5$ half of the sites are culled in the first iteration. This implies, that to obtain a spanning cluster of unculled sites in the $\mathrm{SC}$, the $\rho_{c}^{*}(m)=c_{1} m+c_{2}$ where $c_{1}>1 / 5$ and $c_{2}=1 / 5$. The variation of $\rho_{c}(m)$ with $m$ is studied and shown in Fig. 4 where $c_{1}=0.271$ and $c_{2}=0.198$ are obtained.

We finally study a continuum BPM in terms of percolation of overlaping boxes [14]. In a region of two dimensional continuous space of size $L \times L$ with periodic boundary conditions along both the directions we uniformly distribute $N$ points so that the probability of a point being within the area $d A$ is proportional to $d A$. Every point is situated at the centre of a square box of size $R=1 / 2$ and if two boxes overlap then the two points belong to the same cluster. The neighbour number $n_{i}$ of the point $i$ is the number of points within the associated box including the point itself. All points with $n_{i}<m$ are culled and the culling process is repeated till a SC is reached which cannot be reduced any further (fig. 5).

To calculate the NNs using a local search method we consider an underlying square lattice of size $L$. A primitive cell of this lattice is referred by its bottom-left integer coordinates $(a, b)$. The serial numbers of all points $N_{a b}$ within the cell $(a, b)$ are stored in an auxiliary array $A(a, b, k)$ with $k=1, N_{a b}$ and $A(a, b, 0)=N_{a b}$. For a point $i$ within the cell 
$(a, b)$, the nine cells from $a-1$ to $a+1$ and from $b-1$ to $b+1$ are searched to pick up $n_{i}$ neighbouring points. In a second auxiliary array $\operatorname{neb}(N, M)$ the serial numbers of $n_{i}$ points are stored in the locations neb $(i, k), k=1, n_{i}$ and $n e b(i, 0)$ stores $n_{i}$.

Culling clusters are initiated from all sites with neb $i, 0)<m$. When a point $i$ is culled, we update $n e b(i, 0)=n_{o}$, unculled neighbours $j$ of $i$ are updated as neb $(j, 0)=n e b(j, 0)-1$ and those with $n e b(j, 0)<m$ are culled in the next time step. A SC is shown in Fig. 5.

The percolation threshold $\rho_{c}^{*}(m)$ and probability $Q_{m}(\rho)$ are similarly defined w.r.t. the average initial density $\rho$ of points. Here again we find a gap in $Q_{m}(\rho)$. Variation of $Q_{m}(\rho)$ is continuous and for different system sizes, curves pass through a fixed point. We studied the case of $m=6$, and the fixed point is found to be around $(10.55,4.65)$. For this model we could go only up to the system size $L=160$ and $\nu \approx 1.4$ and $\beta \approx 0.05$ are obtained. We believe that this continuum bootstrap percolation percolation model belongs to the same universality class as the lattice bootstrap percolation defined above with multiple particles allowed per site, and both of these are in same universality class as the ordinary percolation.

To summarize, we observed that the sequence of culling sites in the BPM is irrelevant and the same stable configuration is obtained irrespective of the particular culling sequence used. This knowledge helps us to devise an efficient algorithm to find out the percolation threshold. We define a generalization of the bootsrap percolation model which allows lattice sites to be occupied by more than one particle. This is the discretization of the natural continuum version of percolation. Unlike the earlier studied bootstrap percolation models which undergo a first order transion for some $m$, we argued, and presented numerical evidence that the percolation transition for this model is continuous for all $m$, and is in the same universality class of usual percolation.

We acknowledge with thanks D. Dhar for many useful discussions and suggestions. I also thankfully acknowledge D. Stauffer, J. Adler and J. R. Banavar for the critical reading of the manuscript. 


\section{Figure Captions:}

1. Starting from a fully occupied square lattice of size $L=32$ with p. b. c., 77 sites (shown by filled squares) are deleted one after the other. Culled clusters of $m=3$ BPM are grown from the deleted sites. The paths of the propagation of the cascading process is shown by lines. The 77-th cluster had grown from the encircled site which evacuates the whole system. This implies that the $p_{c}^{*}(3)=(1-77 / 1024) \approx 0.925$ for this configuration.

2. The percolation probability $Q_{2}(\rho)$ for the generalised BPM with $m=2$ is plotted with the average density $\rho$ for system sizes $L=256$ (circle), 512 (square) and 1024 (triangles). The common point of the three curves is $\left(\rho_{c}^{*}(2), Q_{2}\left(\rho_{c}^{*}(2)\right)\right)=(0.899,0.179)$.

3. The collapse of $\left(Q_{2}(\rho)-Q_{2}\left(\rho_{c}^{*}(2)\right)\right) L^{0.075}$ as a function of $\left(\rho-\rho_{c}^{*}(2)\right) L^{0.75}$ is shown using same symbols for the same system sizes as in Fig. 2.

4. For the generalised BPM, the percolation threshold $\rho_{c}(m)$ is plotted for various $m$ values. The continuous line is a linear fit with slope 0.271 and intercept 0.198 .

5. The stable configuration of continuum BPM with $m=3$ using the initial density of $\rho$ $=6.12$ points per unit area in a system of size $20 \times 20$. Every point is at the centre of a box of size $R=1 / 2$. The boxes belonging to the infinite cluster are shown by filled squares where as those of isolated clusters are shown by open squares. 


\section{References:}

1. J. Chalupa, P. L. Leath and G. R. Reich, J. Phys. C., 12, L31 (1979).

2. P. M. Kogut anf P. L. Leath, 12, 3187 (1981).

3. J. Adler, Physica A, 171, 453 (1991), J. Adler and A. Aharony, J. Phys. A. 211387 (1988), J. A. M. S. Duarte, Physica A, 158, 1075 (1989).

4. M. Pollak and I. Riess, Phys. Status Solidi B69, K15 (1975).

5. D. Stauffer, Introduction to Percolation Theory, (Taylor \& Francis, London), 1985.

6. R. H. Schonmann, J. Stat. Phys. 58, 1239 (1990).

7. J. Straley, unpublished results, presented in [2].

8. J. Adler, D. Stauffer and A. Aharony, J. Phys. A. 22 L297 (1989).

9. S. S. Manna, D. Stauffer and D. W. Heermann, Physica A 16220 (1989).

10. D. Dhar, Phys. Rev. Lett. 64, 1613 (1990).

11. S. S. Manna, J. Stat. Phys. 59, 509 (1990).

12. J. Adler, R. Gross and R. Warmund, Physica A 163, 440 (1990).

13. J. Hoshen and R. Kopelman, Phys. Rev. B., 14, 3428 (1976)

14. R. Meester and R. Roy, Continuum Percolation, Cambridge University Press, 1996. 


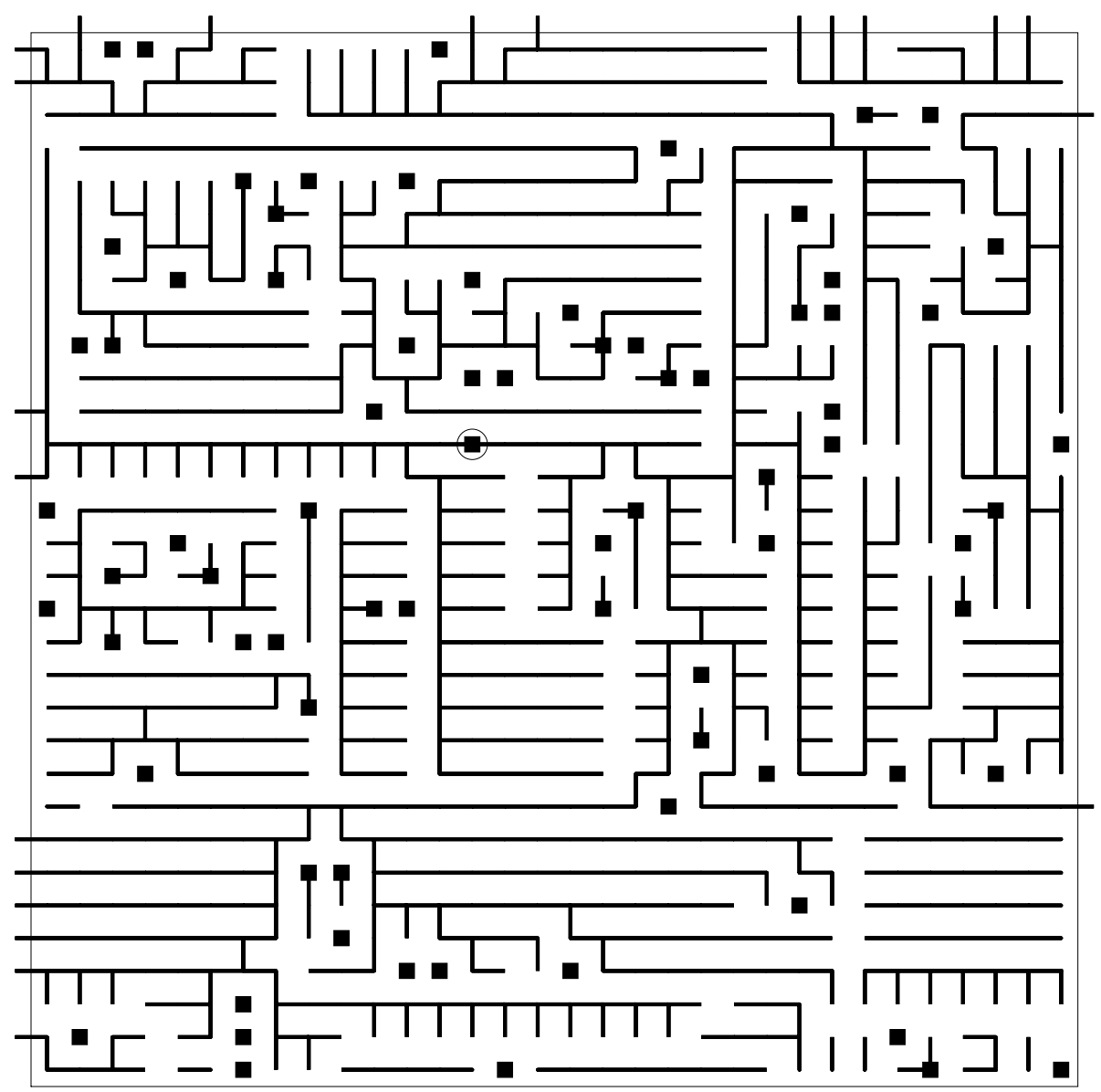

Fig. 1 : S. S. Manna 


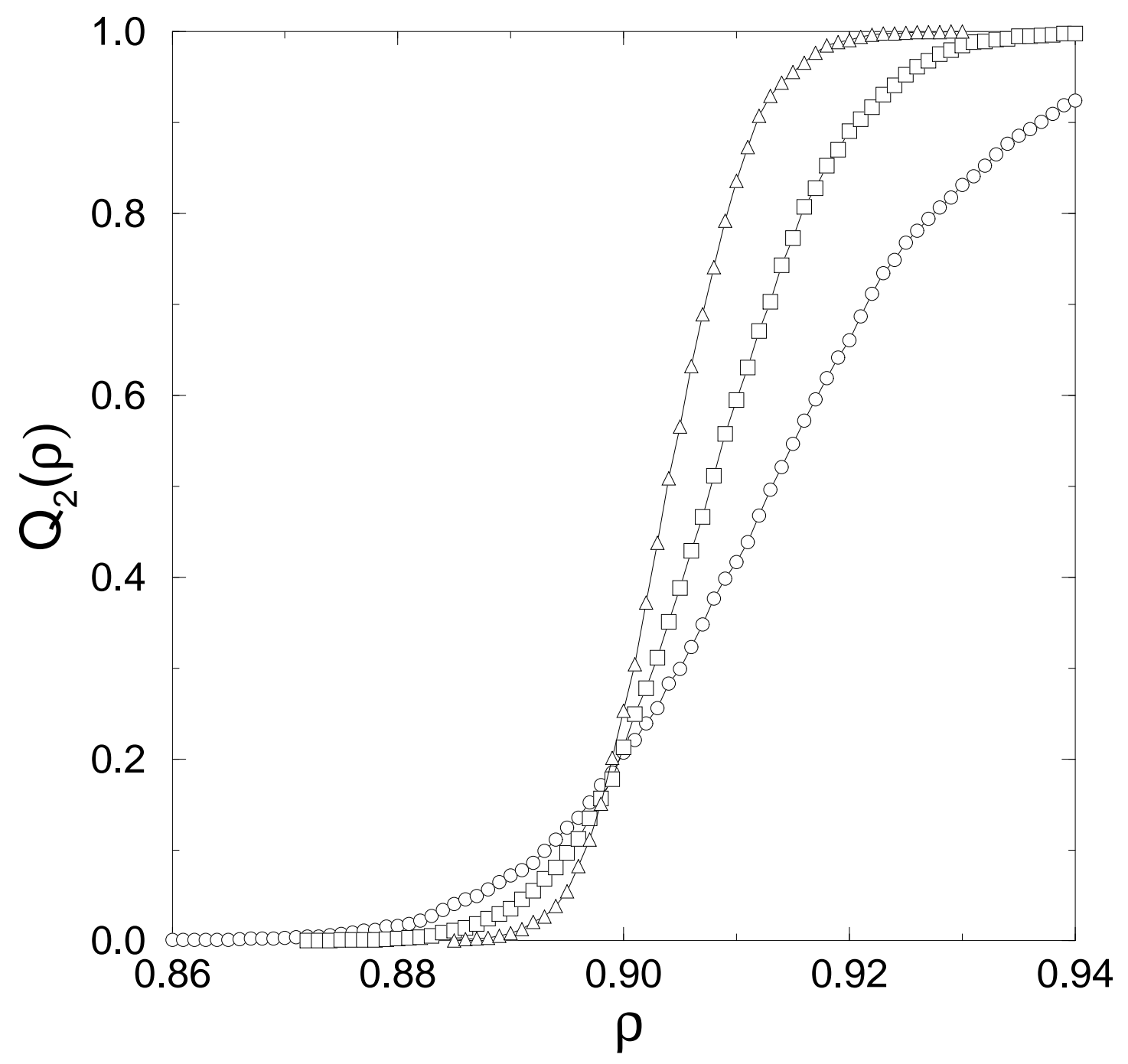

Fig. 2 : S. S. Manna 


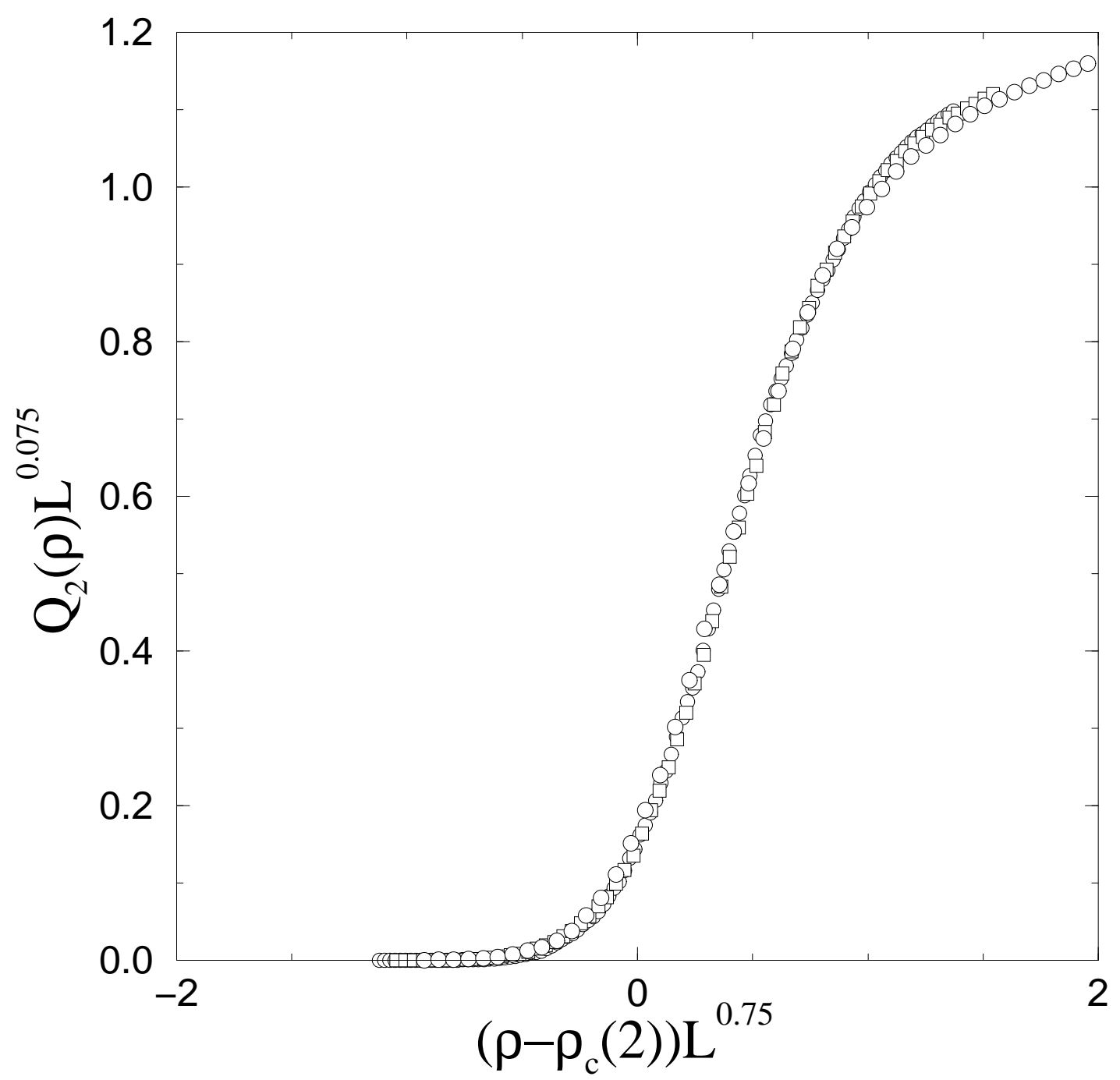

Fig. 3 : S. S. Manna 


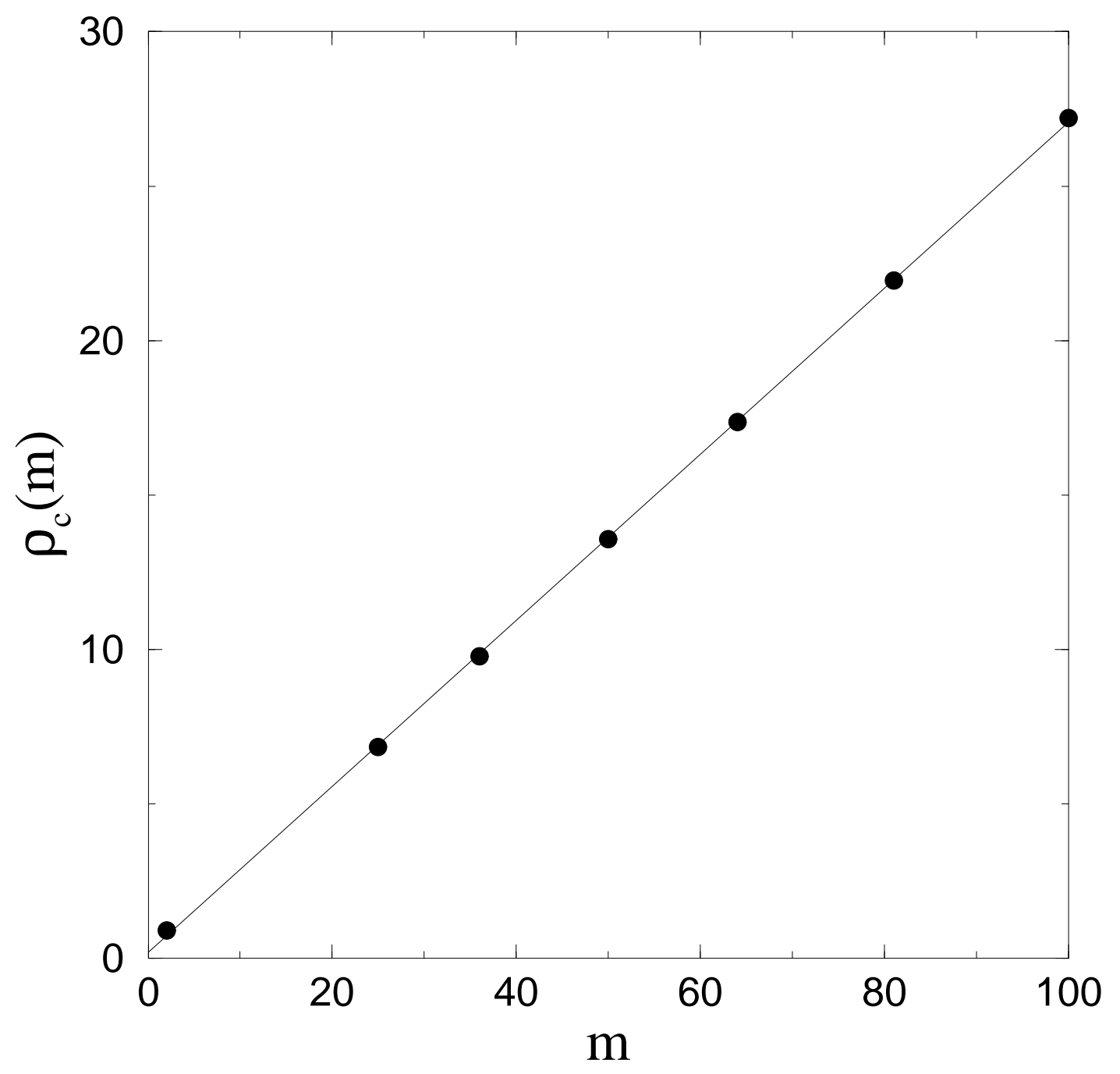

Fig. 4 : S. S. Manna 


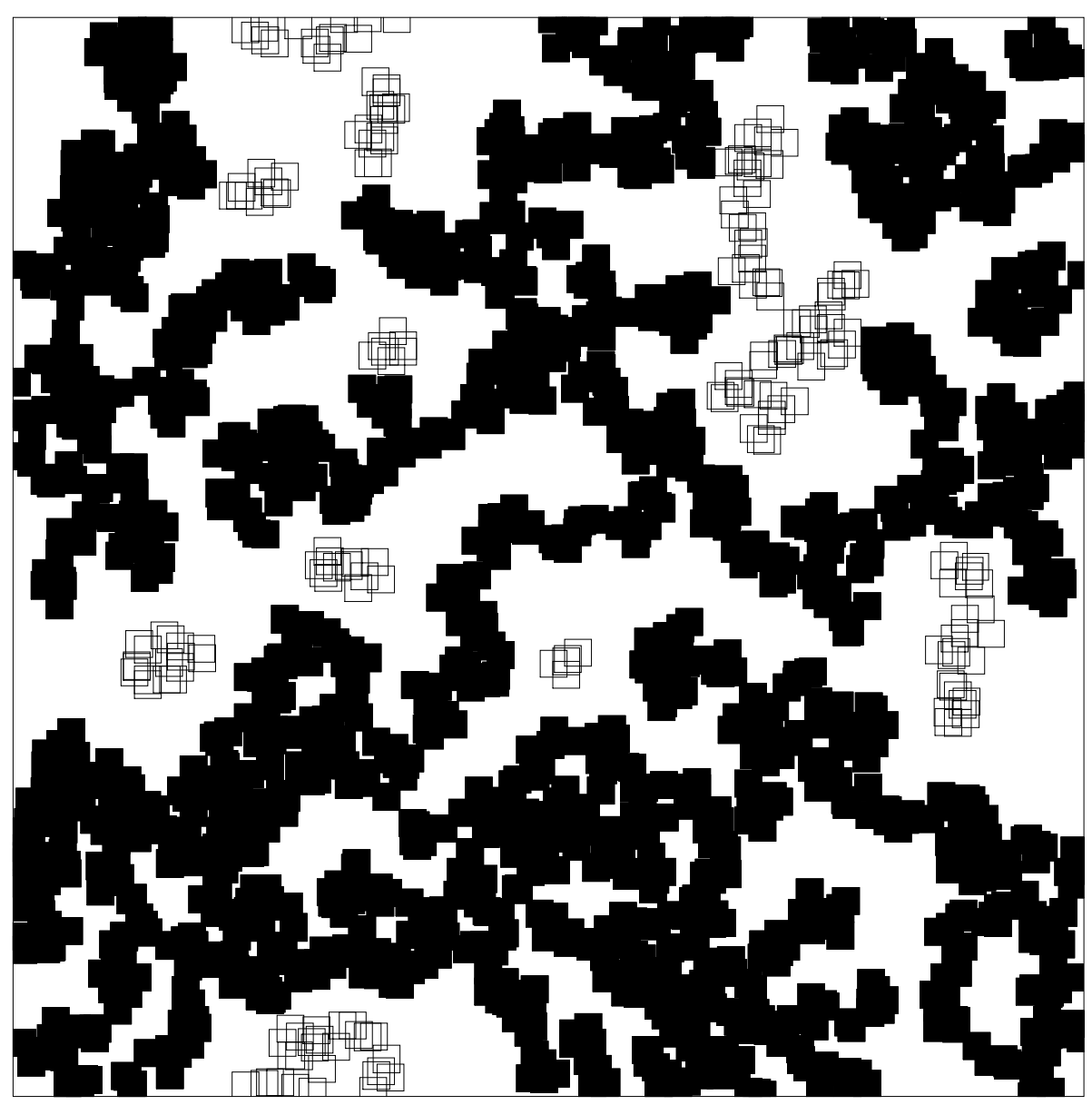

Fig. 5 : S. S. Manna 